OPEN ACCESS

Edited by:

Lawrence Todd Reiter,

University of Tennessee Health

Science Center (UTHSC),

United States

Reviewed by:

Charles D. Nichols,

Louisiana State University,

United States

Brian Staveley,

Memorial University of Newfoundland,

Canada

Maria Claudia Gonzalez Deniselle, CONICET Instituto de Biología y Medicina Experimental (IBYME),

Argentina

*Correspondence:

Scott A. Barbee

scott.barbee@du.edu

Specialty section:

This article was submitted to Genetics of Common and Rare

Diseases,

a section of the journal

Frontiers in Genetics

Received: 20 August 2021 Accepted: 28 September 2021

Published: 12 October 2021

Citation:

Wilkinson EC, Starke EL and Barbee SA (2021) Vps54 Regulates Lifespan and Locomotor Behavior in

Adult Drosophila melanogaster.

Front. Genet. 12:762012.

doi: 10.3389/fgene.2021.762012

\section{Vps54 Regulates Lifespan and Locomotor Behavior in Adult Drosophila melanogaster}

\author{
Emily C. Wilkinson ${ }^{1}$, Emily L. Starke ${ }^{1}$ and Scott A. Barbee ${ }^{1,2 *}$ \\ ${ }^{1}$ Department of Biological Sciences, University of Denver, Denver, CO, United States, ${ }^{2}$ Molecular and Cellular Biophysics \\ Program, University of Denver, Denver, CO, United States
}

Vps54 is an integral subunit of the Golgi-associated retrograde protein (GARP) complex, which is involved in tethering endosome-derived vesicles to the trans-Golgi network (TGN). A destabilizing missense mutation in Vps54 causes the age-progressive motor neuron (MN) degeneration, muscle weakness, and muscle atrophy observed in the wobbler mouse, an established animal model for human MN disease. It is currently unclear how the disruption of Vps54, and thereby the GARP complex, leads to MN and muscle phenotypes. To develop a new tool to address this question, we have created an analogous model in Drosophila by generating novel loss-of-function alleles of the fly Vps54 ortholog (scattered/scat). We find that null scat mutant adults are viable but have a significantly shortened lifespan. Like phenotypes observed in the wobbler mouse, we show that scat mutant adults are male sterile and have significantly reduced body size and muscle area. Moreover, we demonstrate that scat mutant adults have significant ageprogressive defects in locomotor function. Interestingly, we see sexually dimorphic effects, with scat mutant adult females exhibiting significantly stronger phenotypes. Finally, we show that scat interacts genetically with rab11 in MNs to control age-progressive muscle atrophy in adults. Together, these data suggest that scat mutant flies share mutant phenotypes with the wobbler mouse and may serve as a new genetic model system to study the cellular and molecular mechanisms underlying MN disease.

Keywords: Vps54, MN disease, wobbler mice, membrane traffic, drosophila

\section{INTRODUCTION}

Neurodegenerative diseases are severe and often fatal disorders associated with reduced function, or loss of function, of neurological components. This degeneration commonly leads to cognitive impairment and/or motor dysfunction. The primary risk factor associated with neurodegeneration is aging, and as a great portion of the population continues to age the prevalence of such disorders continues to increase (Niccoli and Partridge, 2012). The identification of mutations linked to human neurodegenerative diseases have highlighted several important intracellular pathways that are involved in disease pathogenesis. Many of these genes can be categorized by their contribution to critical intracellular processes including RNA and protein metabolism, axonal and cytoskeletal dynamics, and membrane trafficking (Taylor et al., 2016).

Endocytic trafficking has been implicated in several specialized processes in neurons including axon guidance and outgrowth, synaptic plasticity, and axonal transport (Wojnacki and Galli, 2016). Disruption of pathways involved in the function of endocytic trafficking has been linked to 
progressive neurodegenerative disorders such as amyotrophic lateral sclerosis (ALS), Parkinson's disease (PD), and hereditary spastic paraplegias (HSPs) (Schreij et al., 2016). MN axons appear to be particularly sensitive to mutations in genes involved in membrane trafficking, specifically ALS and HSPs. The membrane trafficking genes that have been implicated in ALS include Alsin (ALS2), C9ORF72, and Optineurin (OPTN) (Hadano et al., 2001; Yang et al., 2001; Devon et al., 2006; Maruyama et al., 2010; Stepto et al., 2014; Waite et al., 2014). Genes involved in HSPs are Spastin (SPG4), Strumpellin (SPG8), Spatacsin (SPG11), Spastizin (SPG15), AP5 (SPG48), and Vps37A (SPG53) (Hazan et al., 1999; Patel et al., 2002; Valdmanis et al., 2007; Hanein et al., 2008; Slabicki et al., 2010; Zivony-Elboum et al., 2012).

A destabilizing missense mutation in the gene encoding for the vacuolar protein sorting-associated protein 54 (Vps54) causes age-progressive MN degeneration in mice. This mouse model, known as the "wobbler" mouse, is used to model human MN disease because it shares many striking phenotypic similarities with ALS (Moser et al., 2013). Vps54 is a core subunit of the heterotetrametric Golgi-associated retrograde protein (GARP) complex and is involved in tethering retrograde transport carriers, derived from endosomes to the trans-Golgi network (TGN) (Bonifacino and Hierro, 2011). The subunits that compose the primary structure of the GARP complex are Vps51, Vps52, Vps53, and Vps54 (Conibear and Stevens, 2000). Destabilization of Vps54 in the wobbler mouse leads to a compensatory decrease in levels of Vps53 and disruption of the assembly of the GARP complex (Perez-Victoria et al., 2010). The $\mathrm{N}$-terminus of yeast and mammalian Vps54 binds to TGNassociated soluble $\mathrm{N}$-ethylmaleimide-sensitive fusion protein attachment protein receptors (t-SNAREs) while the C-terminus interacts with endosomes (Quenneville et al., 2006; Perez-Victoria and Bonifacino, 2009). Knockdown of Vps54 and other GARP complex subunits results in defects in retrograde and anterograde vesicle transport (Conibear and Stevens, 2000; PerezVictoria et al., 2008; Perez-Victoria and Bonifacino, 2009; Hirata et al., 2015). Additionally, knockdown of GARP complex subunits causes lysosomal dysfunction (Perez-Victoria et al., 2008; Perez-Victoria and Bonifacino, 2009). Taken together, these data strongly suggest that Vps54 (and the GARP complex) plays a conserved and essential role in endolysosomal trafficking pathways.

Drosophila melanogaster have a single ortholog of Vps54 called scattered or scat. We have previously shown that disruption of scat causes defects in the development of the Drosophila larval neuromuscular junction (NMJ) (Patel et al., 2020). Moreover, we found that presynaptic scat interacts genetically with $r a b 7$ to regulate the composition of the postsynaptic density via an unknown trans-synaptic mechanism (Patel et al., 2020). We hypothesized that these changes at the larval NMJ may precede neurodegenerative phenotypes in aging adults. Here we demonstrate that loss of scat expression leads to a significant reduction in adult lifespan. We show that scat mutants have sex-specific defects in lifespan, body size, and muscle mass with females exhibiting a more severe phenotype. Female scat mutants also exhibit neurological dysfunction (seizure) and age-progressive defects in locomotor behavior. Finally, we demonstrate that the simultaneous MNspecific disruption of scat expression and rab11 function exacerbates muscle atrophy in adult females, suggesting phenotypes are due to a trafficking defect. These data suggest that the scat loss-of-function model shares many phenotypes with the wobbler mouse, making it a useful tool to study the mechanisms underlying $\mathrm{MN}$ disease.

\section{MATERIALS AND METHODS}

\section{Drosophila Lines and Genetics}

The following fly lines were obtained from the Bloomington Drosophila Stock Center: scat ${ }^{1} \mathrm{cn}^{1}$, C380-Gal4, UAS-Scat ${ }^{\text {TRiP }}$ (HMS01910), UAS-LUC.VALIUM10, UAS-YFP:Rab5, UASYFP:Rab5 (S43N), UAS-YFP:Rab7, UAS-YFP:Rab7(T22N), UAS-YFP:Rab11, UAS-YFP:Rab11(S25N). In our hands, the $\mathrm{cn}^{1}$ allele caused significant differences in locomotor assays relative to controls (our unpublished observations). Therefore, $\mathrm{cn}{ }^{1}$ was recombined away from new scat alleles. Three new fly lines were generated by mobilizing the transposable element insertion in the $s c a t^{1}$ allele by introducing the $\Delta 2-3$ transposase into the genome following standard procedures. The two resulting deletion lines $\left(s c a t^{\Delta 244}\right.$ and $\left.s c a t^{\Delta 312}\right)$ and one precise excision line $\left(s c a t^{329 P E}\right)$ were screened by PCR and DNA sequencing. All fly crosses were maintained on standard Bloomington media at $25^{\circ} \mathrm{C}, 65 \%$ humidity, and a $12: 12 \mathrm{~h}$ light-dark cycle. Unless otherwise noted, scat ${ }^{329 P E}$ was used as the control for comparisons. For overexpression and short hairpin RNAi studies, the UAS/GAL4 system was used (Brand and Perrimon, 1993). To co-overexpress transgenes in MNs, single copies of all indicated elements were crossed into a background containing one copy of the GAL4 transgene. The GAL4 line used in this work was the MN-specific driver, C380GAL4 (Budnik et al., 1996).

\section{Longevity Assay}

Male and female flies from each genotype were collected within $24 \mathrm{~h}$ of eclosion and segregated by sex. Populations of 300 flies were used in each cohort. All flies were transferred onto fresh food every 2 days and scored for survival at transfer.

\section{Determination of Gender Ratios, Eclosion, and Quantification of Body Size}

For the quantification of gender ratios, 100 adult flies from each genotype were collected at random over a 24 -h period posteclosion and then sexed. For the determination of eclosion percentages, 50 wandering third instar larvae of each genotype were collected, allowed to pupate, and adult flies collected and sexed. For the analysis of adult body size, 5 adults of each sex and genotype were collected within 24 of eclosion and allowed to age for $24 \mathrm{~h}$. Flies were anesthetized with $\mathrm{CO}_{2}$ and the ventral side of the abdomen was imaged using Leica S9i stereo microscope with 10MP CMOS-camera. Length was determined by drawing and measuring a line from the rostral to caudal ends using the 
measurement tools in open-source Fiji/ImageJ2. Size was determined by drawing a line around the thorax and abdomen and calculating area.

\section{Paraffin Embedding, Sectioning, and Image Analysis}

Flies were collected within $24 \mathrm{~h}$ of eclosion and allowed to age until the desired time point. Flies were anesthetized using $\mathrm{CO}_{2}$ and oriented in a custom $3 \mathrm{D}$ printed embedding collar so that the thorax was oriented towards the blades. Flies in the collar were then incubated overnight at $4^{\circ} \mathrm{C}$ with Carnoy's fixative. Flies were then dehydrated by sequentially incubating for 20 min each in room temperature 40, 70, and $100 \%$ ethanol. Flies were then transferred to a 1:1 solution of methyl benzoate: paraffin wax and incubated for $1 \mathrm{~h}$ at $65^{\circ} \mathrm{C}$. For embedding, flies in the collar were transferred to a foil pocket which was then filled with melted paraffin wax and incubated at $65^{\circ} \mathrm{C}$ for $2 \mathrm{~h}$. Following incubation, the pocket was stored overnight at room temperature to allow the wax to harden. Paraffin embedded tissue was sectioned into $10 \mu \mathrm{m}$ sections using a Leica RM2125 microtome and floated on cold water. Sections were collected using charged glass microscope slides and allowed to dry for $2 \mathrm{~h}$ at room temperature. Slides were deparaffinized by incubation in room temperature xylene for $15 \mathrm{~min}$. Tissue was rehydrated prior to staining by sequentially incubating for $10 \mathrm{~min}$ each in $100,95,80 \%$ ethanol and $\mathrm{ddH}_{2} \mathrm{O}$. Tissue was stained by incubating slides with hematoxylin for $5 \mathrm{~min}$ followed by eosin for $30 \mathrm{~s}$ with to washes in between treatments $\left(\mathrm{diH}_{2} \mathrm{O}\right.$ followed by $95 \%$ ethanol). Tissue was then dehydrated by treating incubating slides for $15 \mathrm{~min}$ each in $95 \%$ then $100 \%$ ethanol and stored in xylene overnight at room temperature. Permount, a xylene based mounting media, was used to mount and store stained sections. Sections were imaged using a Laxco SeBa 2 series digital microscope system with a $10 \mathrm{X}$ objective (N.A. 1.25). Image analysis was done using the measurement tools in Fiji/ImageJ2. Muscle area was determined by tracing around the perimeter of all 12 dorsal longitudinal muscles together excluding any space between individual muscles. Thoracic area was determined by tracing around the cuticle. Sections with less than $\sim 90 \%$ intact cuticle were excluded from analysis.

\section{Spontaneous Flight Assay}

The flight assay was modified slightly from a published protocol (Benzer, 1973). For the analysis of flight behavior, 20 female flies of each genotype were collected within $24 \mathrm{~h}$ of eclosion and allowed to age until the desired time point and briefly stored in an empty containment vial. Flies were lightly tapped to the bottom of the vial and then dropped from a unform height into a funnel on top of a $500 \mathrm{ml}$ graduated cylinder. The inside of the cylinder was coated with a thin layer of mineral oil. When flies enter a free fall, they will attempt to fly to and land on the nearest surface. When flies land on the side of the cylinder they become stuck in the mineral oil. Flies with poor locomotor function are expected to fall farther in the cylinder before landing on the side. The height of the graduated cylinder was separated into equal quadrants. The quadrant at which each fly landed and stuck to the side of the cylinder was recorded for each genotype.

\section{Negative Geotaxis Assay}

For the analysis of climbing behavior, 20 female flies of each genotype were collected within $24 \mathrm{~h}$ of eclosion and allowed to age until the desired time point. Flies tapped to the bottom of a container exhibit a climbing reflex, where they favor climbing over flight to regain position at the top of a container. Flies were transferred to an empty straight walled polystyrene Drosophila vial (Fisher Scientific \#AS515) marked with the target height, gently tapped down to the bottom, and allowed to climb up the sides for $90 \mathrm{~s}$. Because scat mutant flies were generally poor climbers, the target height was set relatively low $(1 \mathrm{~cm})$. Data was recorded using the built-in camera on a MacBook Air and Photo Booth video recording software. All genotypes were tested in triplicate. Data was analyzed manually looking at individual video frames. The number of flies that climbed past the target mark on the vial after $90 \mathrm{~s}$ was recorded.

\section{Bang Sensitivity Assay}

To analyze seizure phenotypes, bang-sensitivity assays were performed essentially as previously described (Song et al., 2008; Kroll and Tanouye, 2013). 100 female flies per genotype were collected within $24 \mathrm{~h}$ after eclosion under $\mathrm{CO}_{2}$ and moved to a fresh vial with media and allowed to recover overnight. For testing, flies were transferred into an empty vial and vortexed at maximum speed for $10 \mathrm{~s}$. The bang-sensitive phenotype was scored as the number of flies that did not experience paralysis or seizure lasting more than $20 \mathrm{~s}$. Flies for each genotype were collected and tested over a period of several days and the results of each experiment pooled.

\section{Statistics}

All data was recorded in Microsoft Excel and processed using Prism 9 (Graphpad). Results were statistically significant at $p<$ 0.05 . Results shown throughout the study are mean \pm SEM. n.s. $=$ not significant, ${ }^{*} p<0.05,{ }^{* *} p<0.01,{ }^{* * *} p<0.001$, and ${ }^{* * *} p<$ 0.0001 . Statistical tests used for each experiment are found in respective figure legends.

\section{RESULTS}

\section{Generation of New scat Alleles}

To examine scat function in adult Drosophila, we first generated new alleles by mobilizing the P-element insertion located in the $5^{\prime}$ end of the $s c a t^{1}$ allele (Figure 1A). scat ${ }^{1}$ is thought to be a protein null that causes male sterility and defects and the larval NMJ (Castrillon et al., 1993; Fari et al., 2016; Patel et al., 2020). We isolated two partial deletions of scat $\left(\right.$ scat $^{\Delta 244}$ and scat $\left.{ }^{\Delta 312}\right)$. The scat $^{\Delta 312}$ allele starts at the P-element insertion and deletes $1571 \mathrm{bp}$ of downstream sequence (Figure 1A). The $s c a t^{\Delta 244}$ allele removes the P-element and a $346 \mathrm{nt}$ fragment upstream of the insertion site that spans an intron-exon junction (Figure 1A). When homozygous, both lines produced viable adults and, as with the original scat ${ }^{1}$ line, males were sterile (data not shown). 

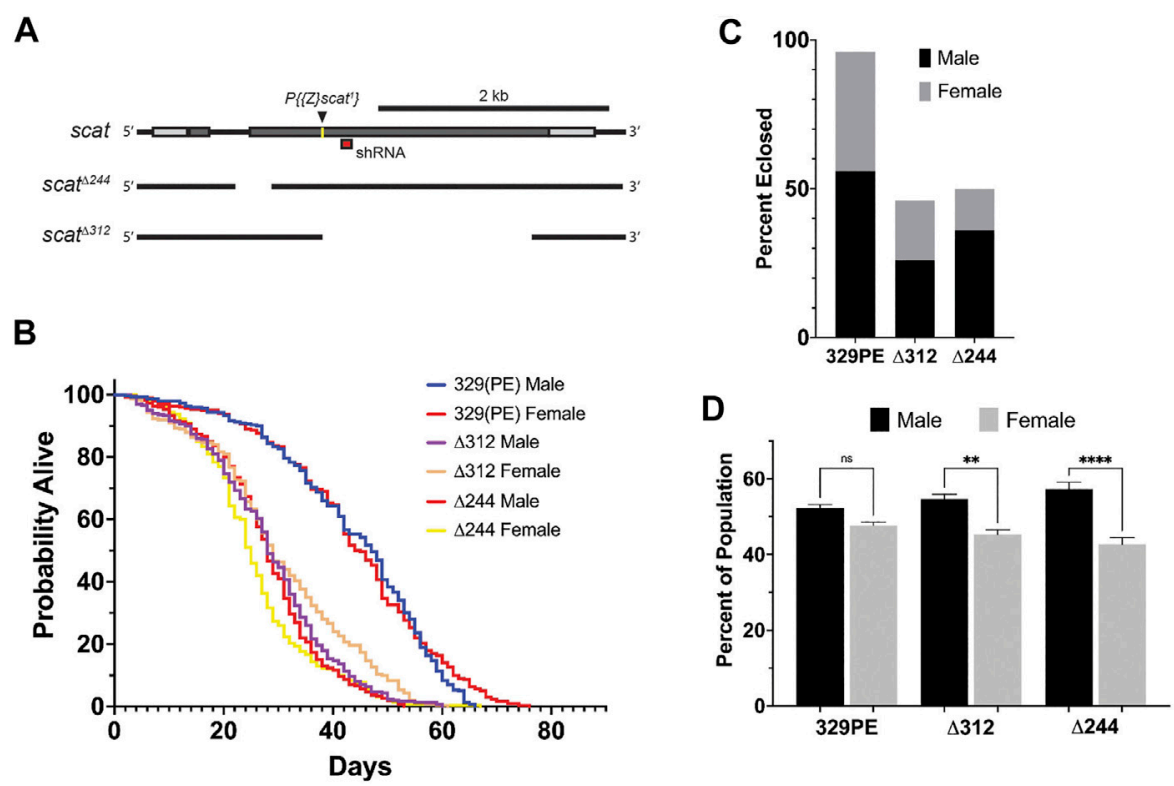

FIGURE 1 | scat mutant adults have a shortened lifespan and a male-biased sex ratio. (A) Schematic representation of the scat gene showing the insertion site for the scat ${ }^{1}$ P-element (arrowhead) and location of the target sequence for the scat shRNA (red box). The location of the deleted sequence in scat ${ }^{\Delta 244}$ and scat ${ }^{\Delta 312}$ are indicated by gaps. (B) Lifespan analysis of homozygous flies of the indicated genotype and separated by sex $(n=300)$. (C) The proportion of homozygous flies of the indicated genotype that survived pupation by $\operatorname{sex}(n=50)$. (D) Sex ratio of flies homozygous for the indicated genotypes $(n=100)$. Statistics: Ordinary one-way ANOVA with Holm-Sidak post-hoc analysis.

TABLE 1 | Lifespan analysis of scat mutants.

Median life (days)

\begin{tabular}{lc}
\hline scat $^{329 P E}$ male & 47 \\
scat $^{329 P E}$ female & 44.5 \\
scat $^{\Delta 244}$ male & 28 \\
scat $^{\Delta 244}$ female & 25 \\
scat $^{\Delta 312}$ male & 28 \\
scat $^{\Delta 312}$ female & 29
\end{tabular}

Difference vs. sex-matched scat ${ }^{329 P E}(\%)$
44.5

$28-58$

$28-58$

65
Significance vs, sex-matched scat ${ }^{329 P E}$
Finally, a genetic control line was generated by precisely excising the P-element $\left(s c a t^{329 P E}\right)$. This line rescued the male sterility phenotype observed with the scat ${ }^{1}$ and mutant alleles.

\section{scat Mutant Adults Have a Shortened Lifespan and Male-Biased Sex Ratios}

Depending on the severity and progression of symptoms, the lifespan of the wobbler mouse can vary from 120 days to up to 1 year (Duchen and Strich, 1968). To determine if scat mutants also exhibited longevity defects, we conducted lifespan studies in adult flies that were homozygous for each scat allele. In contrast to extensive published work in wild-type Drosophila melanogaster, $s c a t^{329 P E}$ females did not live longer than males suggesting there is some effect of genetic background on longevity in scat ${ }^{329 P E}$ females (Figure 1B and Table 1) (Lints et al., 1983). Thus, statistical comparisons in our study have been made to the more genetically similar $s c a t^{329 P E}$ controls. Importantly, the median lifespans of both scat $^{\Delta 244}$ and $s c a t^{\Delta 312}$ males and females were significantly reduced (Table 1). As with scat ${ }^{329 P E}$, only a small (but significant) difference in lifespan was observed between scat ${ }^{\Delta 244}$ and $s c a t^{\Delta 312}$ males and females (Table 1). Together, these data suggest that disruption of scat results in a reduced lifespan.

The scat ${ }^{1}$ allele has been shown to be semi-lethal prior to eclosion (Castrillon et al., 1993). This contrasts with Vps54 lossof-function in mice which causes embryonic lethality (SchmittJohn et al., 2005). Thus, we next determined whether $s c a t^{\Delta 244}$ and scat $t^{\Delta 312}$ caused pupal lethality. As expected, only $50 \%$ of $s c a t^{\Delta 244}$ and $46 \%$ of $s c a t^{\Delta 312}$ pupae survived to eclosion compared to $96 \%$ for $s c a t^{329 P E}$ (Figure 1C). These data suggest that scat loss-offunction causes significant lethality at the pupal stage.

During these experiments, we also noticed that there were more surviving male then female adults (Figure 1C). To analyze this phenotype more closely, we quantified the proportions of eclosing adult flies from each genotype. While the proportion of 

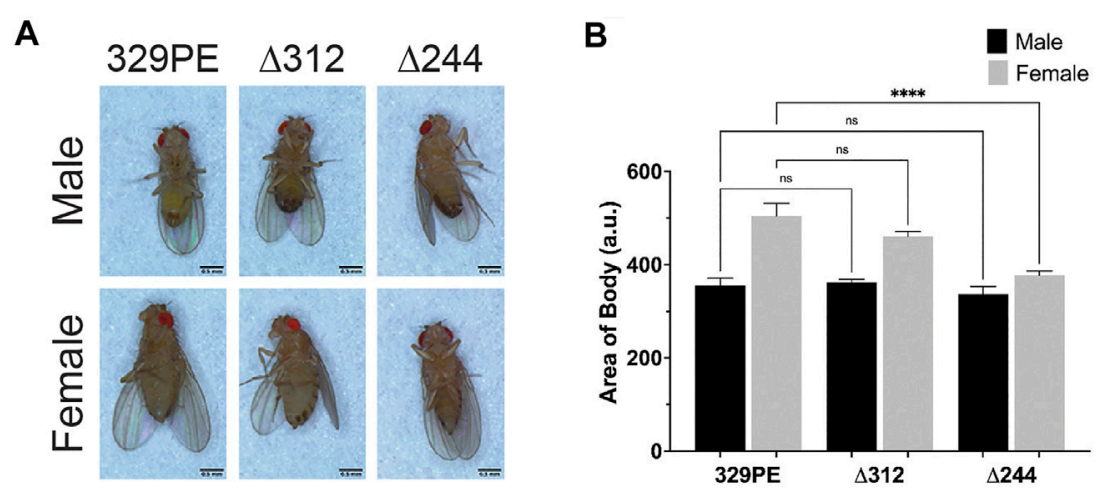

FIGURE 2 | scat mutant females have a reduced body size. (A) Images of representative adult flies of the indicated sex and genotype. Scale bar = 0.5 mm. (B) Quantification of body area of adult flies of the indicated sex and genotype $(n=5)$. Statistics: Ordinary one-way ANOVA with Holm-Sidak post-hoc analysis.

male to female flies $s c a t^{329 P E}$ controls was roughly 1:1, we observed a statistically significant male bias in scat ${ }^{\Delta 244}$ and scat $^{\Delta 312}$ flies (Figure 1D). These results further suggest that adult female flies may be disproportionally affected by loss of scat expression.

\section{scat ${ }^{\wedge 244}$ Mutant Females Have a Significantly Reduced Body Size}

The wobbler mouse has a significantly reduced body size relative to unaffected littermates (Duchen and Strich, 1968). To determine if a similar phenotype was observed in scat mutants, we imaged young adult flies from each genotype to examine body area and length (Figure 2A). Quantification of body area revealed a significant decrease in body size in $s c a t^{\Delta 244}$ females when compared to scat ${ }^{329 P E}$ controls (Figure 2B). No other comparisons were statistically significant. Similarly, the analysis of body length along the longest line drawn from the rostral to caudal ends revealed a statistically significant decrease in scat $^{\Delta 244}$ females (Supplementary Figure S1A). Collectively, these data suggest that disruption of scat in adult females (but not males) results in decreased in body size.

\section{scat Mutant Females Have Neurological and Age-Progressive Locomotor Defects}

In subsequent experiments, we focused on scat mutant females because they exhibit the strongest phenotypes in lifespan, viability, and body size experiments. "Bang sensitive" (bs) behavioral mutants are a means to study tonic-clonic seizures in humans (Song and Tanouye, 2008). Bang sensitivity is a phenotype where affected flies are briefly paralyzed and seize upon receiving a short mechanical shock or "bang" (Benzer, 1971; Ganetzky and Wu, 1982). Many bs mutations are in genes associated with mitochondrial function, shorted lifespan, and age-related neurodegenerative disease (Reynolds, 2018). Each of these phenotypes have also been linked to the wobbler mouse (Duchen and Strich, 1968; Santoro et al., 2004). We conducted experiments to determine if disruption of scat caused bs phenotypes. We found that 2-day old $s c a t^{\Delta 244}$ and $s c a t^{\Delta 312}$ mutant females exhibited robust bs phenotypes (Figure 3A). This suggests that scat mutants are more prone to seizures, although it does not explain how this mechanistically occurs.

Another phenotype associated with the wobbler mouse is an age-progressive motor defect caused by $\mathrm{MN}$ degeneration and muscle atrophy (Duchen and Strich, 1968). Therefore, we next determined whether female scat mutants had analogous locomotor dysfunction. To identify locomotor defects associated with primary muscle groups, we performed a spontaneous flight assay to assess the function of flight muscles. We observed a significant decrease in flight ability in both $s c a t^{\Delta 244}$ and $s c a t^{\Delta 312}$ as early as 1 day following eclosion (Figure 3B). Moreover, while $s c a t^{329 P E}$ showed little change at 2 weeks of age, flight ability in $s c a t^{\Delta 244}$ and $s c a t^{\Delta 312}$ progressively worsened over time (Figure 3B). To further examine adult locomotor ability, we performed a climbing assay. One major benefit of this approach is that it allowed us to examine the same group of females as they aged. While climbing defects were not observed in scat mutants at 1 day of age, significant differences between both scat mutants and controls were observed at 7 days and this became progressively worse at 14 days post-eclosion (Figures 3C-E). Together, these data suggest that scat mutants have age-progressive locomotor defects.

\section{scat Mutant Females Have Reduced Size and Degeneration of Longitudinal Muscles}

Age-related flight and climbing defects observed in scat mutants suggest that there may be muscle dysfunction or degeneration. To address this question, we conducted a histological analysis of the major thoracic muscle in female flies at 1 and 7 days after eclosion. Light micrographs of histological sections revealed that the size and organization of the six bilaterally paired dorsal longitudinal muscles were significantly smaller in scat mutants (Figures 4A-C). In scat ${ }^{\Delta 312}$ females, muscle degeneration was often observed in some longitudinal muscles (Figure 4A; arrow). In contrast, this phenotype was never observed in $s c a t^{329 P E}$ controls. The reduction in muscle area became significantly more pronounced in older flies (Figure 4C). Interestingly, this "compacted muscle" phenotype is most like those observed in 

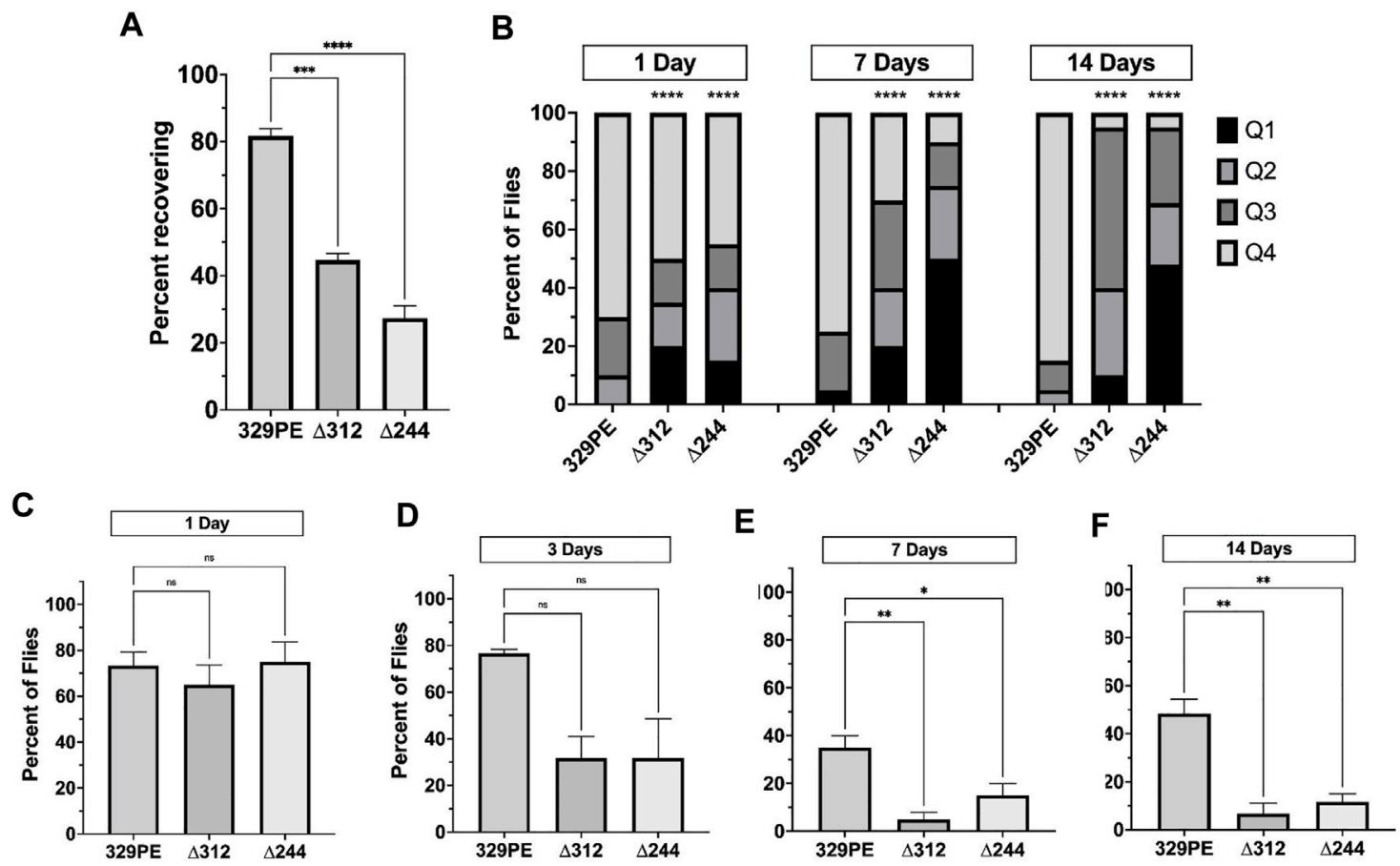

FIGURE 3 | scat mutant females have neurological and age-progressive locomotor defects. (A) Quantification of the number of female flies of the indicated genotypes that recovered within $20 \mathrm{~s}$ following vortexing in bang sensitivity assays $(n=100)$. (B) Quantification of the number of flies that landed in each quadrant in spontaneous flight assays. Q1 indicates the highest quadrant and Q4 is the lowest $(n=20)$. (C-F) Quantification of the number of female flies of the indicated genotypes able to cross the $1 \mathrm{~cm}$ threshold after climbing for $30 \mathrm{~s}(n=20)$ at (C) 1 day, (D) 3 days, (E) 7 days, and (F) 14 days after eclosion. Statistics: (A, C-F) Ordinary oneway ANOVA with Holm-Sidak post-hoc analysis. (B) Chi square analysis compared to 329PE control.
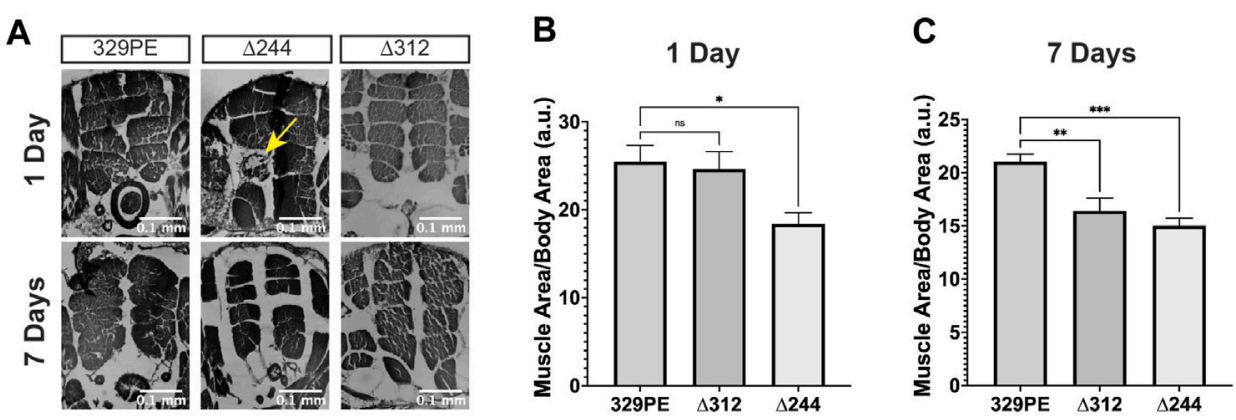

FIGURE 4 | scat mutant females have defects in muscle size and signs of atrophy. (A) Representative H\&E stained thoracic muscle sections of female flies of the indicated age and genotypes. Sections were obtained in the same region of the thorax and oriented so that the dorsal axis is up. Yellow arrows indicate muscle with signs of atrophy. (B, C) Quantification of the muscle area for female flies of the indicated age and genotype $(n=11-20)$. Statistics: Ordinary one-way ANOVA with Holm-Sidak post-hoc analysis.

Drosophila models for myotonic dystrophy type 1 (DM1) (GarciaLopez et al., 2008; Bargiela et al., 2015).

\section{scat Interacts Genetically with Rab11 to Control Locomotion and Muscle Atrophy}

The GARP complex interacts with and regulates the tethering of both early and late endosomes at the TGN (Conboy and Cyert, 2000; Conibear and Stevens, 2000; Siniossoglou and Pelham, 2002;
Conibear et al., 2003). Early and late endosomes are defined and regulated by the small GTPases, Rab5 and Rab7 (respectively). We have previously shown that scat interacts genetically with rab7 (but not with rab5 or rab11) in MNs to regulate synaptic integrity and development at the MNJ in fly larvae (Patel et al., 2020). We hypothesized that this interaction may persist into adulthood. For this analysis, we used an inducible transgenic scat short hairpin RNAi line (UAS-Scat ${ }^{T R i P}$ ) and lines that drive the expression of either wild-type or dominant negative (DN) forms of Rab proteins 

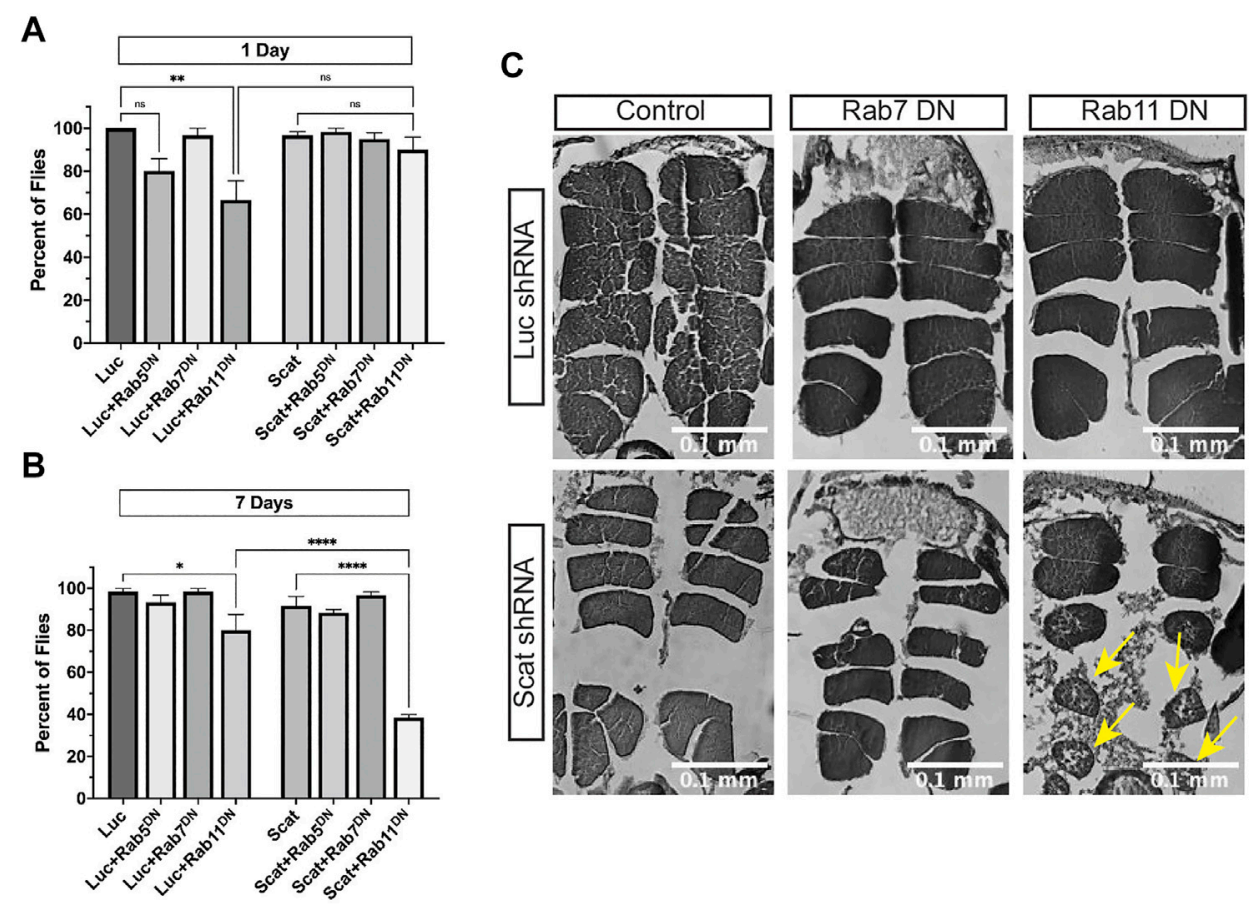

FIGURE 5 | scat interacts genetically with rab11 to control locomotion and muscle integrity. (A, B) Quantification of the number of female flies of the indicated age and genotypes able to cross the $1 \mathrm{~cm}$ threshold after climbing for $30 \mathrm{~s}(n=20)$. Here, expression of either a control or scat shRNA transgene (UAS-Luc shRNA and UAS$s c a t^{\text {shRNA }}$ ) and dominant negative Rab transgene (UAS-rab ${ }^{D N}$ ) was driven in MNs by C380-Ga/4. (C) Representative H\&E stained thoracic muscle sections of female flies of the indicated age and genotypes. Sections were obtained in the same region of the thorax and oriented so that the dorsal axis is up. Yellow arrows indicate muscle with signs of atrophy. Statistics: Ordinary one-way ANOVA with Holm-Sidak post-hoc analysis.

$\left(U A S-R a b^{W T}\right.$ and $\left.U A S-R a b^{D N}\right)$. The scat RNAi and rab DN constructs were used so that we could specifically disrupt expression (or function) of both genes only in MNs.

We first determined if scat interacted genetically with $r a b 5, r a b 7$, and rab11 to regulate age-progressive locomotion in climbing assays. $\mathrm{MN}$-specific overexpression of Rab proteins paired with knockdown of scat had no effect on climbing (Supplementary Figures S2A, B). In contrast, disruption of only rab11 paired with scat RNAi significantly reduced climbing ability of female flies at 7 days post-eclosion (Figure 5B). This was still significant even though disruption of rab11 in controls had a negative effect (Figures 5A,B). Surprisingly, disruption of both scat and $\mathrm{rab} 7$ had no effect on climbing ability after 7 days (Figure 5B). To determine the nature of the locomotor defects, we also examined the major thoracic muscles in histological sections. As with climbing, we observed no significant morphological differences when scat knockdown was paired with the overexpression of wild-type rab7 or rab11 (Supplementary Figure S2C). Strikingly, we saw significant muscle atrophy in most females when both scat and rab11 were disrupted in MNs (Figure 5C). Together, this suggests that scat interacts genetically with rab11 and not $r a b 7$ in adult MNs.

\section{DISCUSSION}

One of the primary objectives of this study was to determine whether disruption of scat expression in adult flies caused phenotypes like those observed in the wobbler mouse. While mutations in Vps54 have not been directly linked to human disease, the wobbler mouse has been used by many researchers as a model for the sporadic form of ALS because of striking similarities to ALS pathology (Ikeda et al., 1998; Dennis and Citron, 2009; Nieto-Gonzalez et al., 2011; Moser et al., 2013; Dahlke et al., 2015; Schmitt-John, 2015; Klatt et al., 2019; Rohm et al., 2019; Cihankaya et al., 2021; De Nicola et al., 2021). It has previously been shown that scat mutants share spermatogenesis defects caused by Golgi dysfunction with the wobbler mouse (Castrillon et al., 1993; Fari et al., 2016). Here, we find that scat mutants share additional phenotypes including locomotor defects, decreased body size, muscle atrophy, and a shortened lifespan (Duchen and Strich, 1968). Importantly, these phenotypes are also consistent with degenerative human $\mathrm{MN}$ diseases, most notably ALS (Bruijn et al., 2004). While not all wobbler phenotypes could be examined here, our data suggests that scat loss- or MN-specific reduction-of-function may serve as a new model to study progressive $\mathrm{MN}$ disease.

There are some notable differences between scat mutant flies and the wobbler mouse. First, loss-of-function of Vps54 in mice causes embryonic lethality characterized by the underdevelopment of cardiac muscle and motor neurons (Schmitt-John et al., 2005). In contrast, loss of scat expression in flies causes only partial lethality, primarily in females. Second, the loss of MNs in the wobbler mouse leads to muscle spasticity and not seizures, as we observe in flies (Duchen and Strich, 1968). 
However, mutations in Vps53 have been linked to the seizure phenotypes observed in pontocerebellar hypoplasia type $2 \mathrm{E}$ (PCH2E) suggesting that seizures may be associated with disruption of the GARP complex (Feinstein et al., 2014). Further work is required to determine if scat mutants exhibit evidence of defects in the brain like those observed in fly models for PCH (Morton et al., 2020). Finally, there is no evidence of phenotypic sexual dimorphism in the wobbler mouse. Moreover, while sex has been reported to be a significant factor influencing ALS development, males have been found to be more susceptible than females (Trojsi et al., 2020). It is likely that our results are due to differences between Drosophila and mammalian neurophysiology. For example, there is a significant amount of evidence suggesting that female-specific steroid hormones like estrogen, that are lacking in flies, have neuroprotective properties (Zarate et al., 2017).

While we do not directly show that disruption of scat causes $\mathrm{MN}$ phenotypes, we provide evidence in support of this hypothesis. We find that knockdown of scat in the presynaptic MN paired with disruption of Rab11 activity significantly reduces locomotor ability of females and causes atrophy in the postsynaptic muscle (Figures 5A-C). Similarly, we have previously shown that presynaptic knockdown of scat combined with disruption of $r a b 7$ in larval MNs disrupts the integrity of postsynaptic densities at the NMJ via an unknown mechanism (Patel et al., 2020). We speculate that neuromuscular dysfunction starts to occur in larvae and begins to manifest as muscle atrophy during metamorphosis and early adulthood (Kuleesha et al., 2016). Muscle atrophy is commonly associated with neurodegenerative disorders involved in the peripheral nervous system such as ALS, PD, multiple sclerosis (MS), and Charcot-Marie-Tooth disease (CMT) (Dyck and Lambert, 1968; Harding and Thomas, 1980; Leger et al., 2006; Allen et al., 2008; Peker et al., 2018). As MNs progressively denervate myofibrils, muscle atrophy occurs, preceded by a decrease in sarcolemma permeability. This most commonly manifests in neuromuscular disorders as muscle weakness and loss of muscle mass (Cisterna et al., 2014). Additional work is required to determine if scat mutants directly cause $\mathrm{MN}$ degeneration.

It has been proposed that wobbler phenotypes are the direct result of defects in endolysosomal trafficking via the disruption of the GARP complex (Perez-Victoria et al., 2010). Our published data showing a genetic interaction between rab7 in larval MNs and with rab11 in adults suggest this mechanism is conserved in Drosophila (Patel et al., 2020). Why is there a transition to rab11 in adults? Rab11 mediates endosome recycling to the TGN and plasma membrane and regulates the function of recycling endosomes (REs) (Kelly et al., 2012). While Vps54 does not interact with REs directly, they are important components involved in vesicular recycling and they a play critical role in axon development, axon pathfinding, synaptic vesicle recycling, and synaptic plasticity (Rozes-Salvador et al., 2020). Levels of rab11 are downregulated in many neurodegenerative diseases including ALS (Zhang et al., 2020). Finally, Rab11 has neuroprotective effects. For example, the overexpression of rab11 in neurons rescues synaptic and locomotor defects in a Drosophila model for Huntington's disease (HD) (Steinert et al., 2012).

In summary, we have provided data suggesting that scat lossof-function flies share phenotypes that are characteristic of the wobbler mouse. Based on this, we propose that this Drosophila model, or "wobbler fly", may serve a new tool to study the mechanisms that underly progressive $\mathrm{MN}$ disease in humans. It would be interesting at this point to determine if scat loss-offunction causes degeneration in adult MNs. Regardless, we can now leverage the power of Drosophila genetics and use this model to identify novel modifiers of the scat locomotor phenotypes followed by detailed characterization of genetic interactors. We have already demonstrated the utility of this model on a focused scale by our analysis of interaction with the $r a b$ genes.

\section{DATA AVAILABILITY STATEMENT}

The raw data supporting the conclusions of this article will be made available by the authors, without undue reservation.

\section{AUTHOR CONTRIBUTIONS}

EW, ES, and SB designed the experiments. EW and ES performed the experiments. EW, ES, and SB analyzed the data. EW wrote the first draft of the manuscript. All authors contributed to manuscript revision, read, and approved the submitted version.

\section{FUNDING}

This work was funded by grants awarded to SB by the University of Denver (DU) and Knoebel Center for Healthy Aging at DU. The funders had no role in the study design, data collection or analysis, decision to publish, or preparation of the manuscript. Some fly strains were obtained from the Bloomington Drosophila Stock Center, which is funded by National Institutes of Health grant P40OD018537.

\section{ACKNOWLEDGMENTS}

We thank S. Van Engelenburg for assistance with developing the fly collar used for histological section. We also thank P. Patel for initial assistance generating scat deletion lines. We thank members of the Barbee lab for providing useful discussion and critical comments.

\section{SUPPLEMENTARY MATERIAL}

The Supplementary Material for this article can be found online at: https://www.frontiersin.org/articles/10.3389/fgene.2021.762012/ full\#supplementary-material 


\section{REFERENCES}

Allen, J. A., Stein, R., Baker, R. A., and Royden Jones, H. (2008). Muscle Atrophy Associated with Multiple Sclerosis: a Benign Condition or the Onset of Amyotrophic Lateral Sclerosis? J. Clin. Neurosci. 15, 706-708. doi:10.1016/ j.jocn.2007.04.024

Bargiela, A., Cerro-Herreros, E., Fernandez-Costa, J. M., Vilchez, J. J., Llamusi, B., and Artero, R. (2015). Increased Autophagy and Apoptosis Contribute to Muscle Atrophy in a Myotonic Dystrophy Type 1 Drosophila Model. Dis. Model. Mech. 8, 679-690. doi:10.1242/dmm.018127

Benzer, S. (1971). From the Gene to Behavior. JAMA 218, 1015-1022. doi:10.1001/ jama.218.7.1015

Benzer, S. (1973). Genetic Dissection of Behavior. Sci. Am. 229, 24-37. doi:10.1038/ scientificamerican1273-24

Bonifacino, J. S., and Hierro, A. (2011). Transport According to GARP: Receiving Retrograde Cargo at the Trans-golgi Network. Trends Cel Biol. 21, 159-167. doi:10.1016/j.tcb.2010.11.003

Brand, A. H., and Perrimon, N. (1993). Targeted Gene Expression as a Means of Altering Cell Fates and Generating Dominant Phenotypes. Development 118, 401-415. doi:10.1242/dev.118.2.401

Bruijn, L. I., Miller, T. M., and Cleveland, D. W. (2004). Unraveling the Mechanisms Involved in Motor Neuron Degeneration in ALS. Annu. Rev. Neurosci. 27, 723-749. doi:10.1146/annurev.neuro.27.070203.144244

Budnik, V., Koh, Y.-H., Guan, B., Hartmann, B., Hough, C., Woods, D., et al. (1996). Regulation of Synapse Structure and Function by the Drosophila Tumor Suppressor Gene Dlg. Neuron 17, 627-640. doi:10.1016/s0896-6273(00) 80196-8

Castrillon, D. H., Gönczy, P., Alexander, S., Rawson, R., Eberhart, C. G., Viswanathan, S., et al. (1993). Toward a Molecular Genetic Analysis of Spermatogenesis in Drosophila melanogaster: Characterization of MaleSterile Mutants Generated by Single P Element Mutagenesis. Genetics 135, 489-505. doi:10.1093/genetics/135.2.489

Cihankaya, H., Theiss, C., and Matschke, V. (2021). Little Helpers or Mean RogueRole of Microglia in Animal Models of Amyotrophic Lateral Sclerosis. Int. J. Mol. Sci. 22, 993. doi:10.3390/ijms22030993

Cisterna, B. A., Cardozo, C., and Sãjez, J. C. (2014). Neuronal Involvement in Muscular Atrophy. Front. Cel. Neurosci. 8, 405. doi:10.3389/fncel.2014.00405

Conboy, M. J., and Cyert, M. S. (2000). Luvlp/Rkilp/Tcs3p/Vps54p, a Yeast Protein that Localizes to the Late Golgi and Early Endosome, Is Required for normal Vacuolar Morphology. MBoC 11, 2429-2443. doi:10.1091/mbc.11.7.2429

Conibear, E., Cleck, J. N., and Stevens, T. H. (2003). Vps51p Mediates the Association of the GARP (Vps52/53/54) Complex with the Late Golgi T-SNARE Tlglp. MBoC 14, 1610-1623. doi:10.1091/mbc.e02-10-0654

Conibear, E., and Stevens, T. H. (2000). Vps52p, Vps53p, and Vps54p Form a Novel Multisubunit Complex Required for Protein Sorting at the Yeast Late Golgi. $M B o C$ 11, 305-323. doi:10.1091/mbc.11.1.305

Dahlke, C., Saberi, D., Ott, B., Brand-Saberi, B., Schmitt-John, T., and Theiss, C. (2015). Inflammation and Neuronal Death in the Motor Cortex of the Wobbler Mouse, an ALS Animal Model. J. Neuroinflammation 12, 215. doi:10.1186/ s12974-015-0435-0

De Nicola, A. F., Meyer, M., Garay, L., Kruse, M. S., Schumacher, M., Guennoun, R., et al. (2021). Progesterone and Allopregnanolone Neuroprotective Effects in the Wobbler Mouse Model of Amyotrophic Lateral Sclerosis. Cell Mol Neurobiol. [Epub ahead of print]. doi:10.1007/s10571-021-01118-y

Dennis, J. S., and Citron, B. A. (2009). Wobbler Mice Modeling Motor Neuron Disease Display Elevated Transactive Response DNA Binding Protein. Neuroscience 158, 745-750. doi:10.1016/j.neuroscience.2008.10.030

Devon, R. S., Orban, P. C., Gerrow, K., Barbieri, M. A., Schwab, C., Cao, L. P., et al. (2006). Als2-deficient Mice Exhibit Disturbances in Endosome Trafficking Associated with Motor Behavioral Abnormalities. Proc. Natl. Acad. Sci. 103, 9595-9600. doi:10.1073/pnas.0510197103

Duchen, L. W., and Strich, S. J. (1968). An Hereditary Motor Neurone Disease with Progressive Denervation of Muscle in the Mouse: the Mutant 'wobbler'. J. Neurol. Neurosurg. Psychiatry 31, 535-542. doi:10.1136/jnnp.31.6.535

Dyck, P. J., and Lambert, E. H. (1968). Lower Motor and Primary Sensory Neuron Diseases with Peroneal Muscular Atrophy. Arch. Neurol. 18, 619-625. doi:10.1001/archneur.1968.00470360041003
Fári, K., Takács, S., Ungár, D., and Sinka, R. (2016). The Role of Acroblast Formation during Drosophila Spermatogenesis. Biol. Open 5, 1102-1110. doi:10.1242/bio.018275

Feinstein, M., Flusser, H., Lerman-Sagie, T., Ben-Zeev, B., Lev, D., Agamy, O., et al. (2014). VPS53mutations Cause Progressive Cerebello-Cerebral Atrophy Type 2 (PCCA2). J. Med. Genet. 51, 303-308. doi:10.1136/jmedgenet-2013101823

Ganetzky, B., and Wu, C.-F. (1982). Indirect Suppression Involving Behavioral Mutants with Altered Nerve Excitability in DROSOPHILA MELANOGASTER. Genetics 100, 597-614. doi:10.1093/genetics/100.4.597

Garcia-Lopez, A., Monferrer, L., Garcia-Alcover, I., Vicente-Crespo, M., AlvarezAbril, M. C., and Artero, R. D. (2008). Genetic and Chemical Modifiers of a CUG Toxicity Model in Drosophila. PLoS One 3, e1595. doi:10.1371/ journal.pone.0001595

Hadano, S., Hand, C. K., Osuga, H., Yanagisawa, Y., Otomo, A., Devon, R. S., et al. (2001). Erratum: A Gene Encoding a Putative GTPase Regulator Is Mutated in Familial Amyotrophic Lateral Sclerosis 2. Nat. Genet. 29, 352. doi:10.1038/ ng1101-352a

Hanein, S., Martin, E., Boukhris, A., Byrne, P., Goizet, C., Hamri, A., et al. (2008). Identification of the SPG15 Gene, Encoding Spastizin, as a Frequent Cause of Complicated Autosomal-Recessive Spastic Paraplegia, Including Kjellin Syndrome. Am. J. Hum. Genet. 82, 992-1002. doi:10.1016/j.ajhg.2008.03.004

Harding, A. E., and Thomas, P. K. (1980). The Clinical Features of Hereditary Motor and Sensory Neuropathy Types I and II. Brain 103, 259-280. doi:10.1093/brain/103.2.259

Hazan, J., Fonknechten, N., Mavel, D., Paternotte, C., Samson, D., Artiguenave, F. et al. (1999). Spastin, a New AAA Protein, Is Altered in the Most Frequent Form of Autosomal Dominant Spastic Paraplegia. Nat. Genet. 23, 296-303. doi: $10.1038 / 15472$

Hirata, T., Fujita, M., Nakamura, S., Gotoh, K., Motooka, D., Murakami, Y., et al. (2015). Post-golgi Anterograde Transport Requires GARP-dependent Endosome-To-TGN Retrograde Transport. MBoC 26, 3071-3084. doi:10.1091/mbc.E14-11-1568

Ikeda, K., Iwasaki, Y., and Kinoshita, M. (1998). Neuronal Nitric Oxide Synthase Inhibitor, 7-nitroindazole, Delays Motor Dysfunction and Spinal Motoneuron Degeneration in the Wobbler Mouse. J. Neurol. Sci. 160, 9-15. doi:10.1016/ s0022-510x(98)00224-x

Kelly, E. E., Horgan, C. P., and McCaffrey, M. W. (2012). Rab11 Proteins in Health and Disease. Biochem. Soc. Trans. 40, 1360-1367. doi:10.1042/BST20120157

Klatt, C. L., Theis, V., Hahn, S., Theiss, C., and Matschke, V. (2019). Deregulated miR-29b-3p Correlates with Tissue-specific Activation of Intrinsic Apoptosis in an Animal Model of Amyotrophic Lateral Sclerosis. Cells 8, 1077. doi:10.3390/ cells8091077

Kroll, J. R., and Tanouye, M. A. (2013). Rescue Ofeasily Shockedmutant Seizure Sensitivity Indrosophilaadults. J. Comp. Neurol. 521, 3500-3507. doi:10.1002/ cne. 23364

Kuleesha, Y., Puah, W. C., and Wasser, M. (2016). A Model of Muscle Atrophy Based on Live Microscopy of Muscle Remodelling in Drosophila Metamorphosis. R. Soc. Open Sci. 3, 150517. doi:10.1098/rsos.150517

Léger, B., Vergani, L., Sorarù, G., Hespel, P., Derave, W., Gobelet, C., et al. (2006). Human Skeletal Muscle Atrophy in Amyotrophic Lateral Sclerosis Reveals a Reduction in Akt and an Increase in Atrogin-1. FASEB J. 20, 583-585. doi:10.1096/fj.05-5249fje

Lints, F. A., Bourgois, M., Delalieux, A., Stoll, J., and Lints, C. V. (1983). Does the Female Life Span Exceed that of the Male. Gerontology 29, 336-352. doi:10.1159/000213136

Maruyama, H., Morino, H., Ito, H., Izumi, Y., Kato, H., Watanabe, Y., et al. (2010). Mutations of Optineurin in Amyotrophic Lateral Sclerosis. Nature 465, 223-226. doi:10.1038/nature08971

Morton, D. J., Jalloh, B., Kim, L., Kremsky, I., Nair, R. J., Nguyen, K. B., et al. (2020). A Drosophila Model of Pontocerebellar Hypoplasia Reveals a Critical Role for the RNA Exosome in Neurons. Plos Genet. 16, e1008901. doi:10.1371/ journal.pgen.1008901

Moser, J. M., Bigini, P., and Schmitt-John, T. (2013). The Wobbler Mouse, an ALS Animal Model. Mol. Genet. Genomics 288, 207-229. doi:10.1007/s00438-0130741-0

Niccoli, T., and Partridge, L. (2012). Ageing as a Risk Factor for Disease. Curr. Biol. 22, R741-R752. doi:10.1016/j.cub.2012.07.024 
Nieto-Gonzalez, J. L., Moser, J., Lauritzen, M., Schmitt-John, T., and Jensen, K. (2011). Reduced GABAergic Inhibition Explains Cortical Hyperexcitability in the Wobbler Mouse Model of ALS. Cereb. Cortex 21, 625-635. doi:10.1093/ cercor/bhq134

Patel, H., Cross, H., Proukakis, C., Hershberger, R., Bork, P., Ciccarelli, F. D., et al. (2002). SPG20 Is Mutated in Troyer Syndrome, an Hereditary Spastic Paraplegia. Nat. Genet. 31, 347-348. doi:10.1038/ng937

Patel, P. H., Wilkinson, E. C., Starke, E. L., McGimsey, M. R., Blankenship, J. T., and Barbee, S. A. (2020). Vps54 Regulates Drosophila Neuromuscular junction Development and Interacts Genetically with Rab7 to Control Composition of the Postsynaptic Density. Biol. Open 9, bio053421. doi:10.1242/bio.053421

Peker, N., Donipadi, V., Sharma, M., McFarlane, C., and Kambadur, R. (2018). Loss of Parkin Impairs Mitochondrial Function and Leads to Muscle Atrophy. Am. J. Physiol. Cell Physiol. 315, C164-C185. doi:10.1152/ajpcell.00064.2017

Perez-Victoria, F. J., Abascal-Palacios, G., Tascon, I., Kajava, A., Magadan, J. G., Pioro, E. P., et al. (2010). Structural Basis for the Wobbler Mouse Neurodegenerative Disorder Caused by Mutation in the Vps54 Subunit of the GARP Complex. Proc. Natl. Acad. Sci. 107, 12860-12865. doi:10.1073/pnas.1004756107

Pérez-Victoria, F. J., Mardones, G. A., and Bonifacino, J. S. (2008). Requirement of the Human GARP Complex for Mannose 6-phosphate-receptor-dependent Sorting of Cathepsin D to Lysosomes. MBoC 19, 2350-2362. doi:10.1091/ mbc.E07-11-1189

Pe'rez-Victoria, F. J., and Bonifacino, J. S. (2009). Dual Roles of the Mammalian GARP Complex in Tethering and SNARE Complex Assembly at the Trans -Golgi Network. Mol. Cel Biol. 29, 5251-5263. doi:10.1128/MCB.00495-09

Quenneville, N. R., Chao, T.-Y., McCaffery, J. M., and Conibear, E. (2006). Domains within the GARP Subunit Vps54 Confer Separate Functions in Complex Assembly and Early Endosome Recognition. MBoC 17, 1859-1870. doi:10.1091/mbc.e05-11-1002

Reynolds, E. R. (2018). Shortened Lifespan and Other Age-Related Defects in Bang Sensitive Mutants of Drosophila melanogaster. G3 (Bethesda) 8, 3953-3960. doi:10.1534/g3.118.200610

Rohm, M., May, C., Marcus, K., Steinbach, S., Theis, V., Theiss, C., et al. (2019). The microRNA miR-375-3p and the Tumor Suppressor NDRG2 Are Involved in Sporadic Amyotrophic Lateral Sclerosis. Cell Physiol. Biochem. 52, 1412-1426. doi:10.33594/000000099

Rozés-Salvador, V., González-Billault, C., and Conde, C. (2020). The Recycling Endosome in Nerve Cell Development: One Rab to Rule Them All? Front Cel Dev. Biol. 8, 603794. doi:10.3389/fcell.2020.603794

Santoro, B., Bigini, P., Levandis, G., Nobile, V., Biggiogera, M., Botti, F., et al. (2004). Evidence for Chronic Mitochondrial Impairment in the Cervical Spinal Cord of a Murine Model of Motor Neuron Disease. Neurobiol. Dis. 17, 349-357. doi:10.1016/j.nbd.2004.07.003

Schmitt-John, T., Drepper, C., Mussmann, A., Hahn, P., Kuhlmann, M., Thiel, C., et al. (2005). Mutation of Vps54 Causes Motor Neuron Disease and Defective Spermiogenesis in the Wobbler Mouse. Nat. Genet. 37, 1213-1215. doi:10.1038/ ng1661

Schmitt-John, T. (2015). VPS54 and the Wobbler Mouse. Front. Neurosci. 9, 381. doi:10.3389/fnins.2015.00381

Schreij, A. M. A., Fon, E. A., and McPherson, P. S. (2016). Endocytic Membrane Trafficking and Neurodegenerative Disease. Cell. Mol. Life Sci. 73, 1529-1545. doi:10.1007/s00018-015-2105-X

Siniossoglou, S., and Pelham, H. R. B. (2002). Vps51p Links the VFT Complex to the SNARE Tlg1p. J. Biol. Chem. 277, 48318-48324. doi:10.1074/ jbc.M209428200

Song, J., Parker, L., Hormozi, L., and Tanouye, M. A. (2008). DNA Topoisomerase I Inhibitors Ameliorate Seizure-like Behaviors and Paralysis in a Drosophila Model of Epilepsy. Neuroscience 156, 722-728. doi:10.1016/j.neuroscience.2008.07.024

Song, J., and Tanouye, M. (2008). From Bench to Drug: Human Seizure Modeling Using Drosophila. Prog. Neurobiol. 84, 182-191. doi:10.1016/ j.pneurobio.2007.10.006
Steinert, J. R., Campesan, S., Richards, P., Kyriacou, C. P., Forsythe, I. D., and Giorgini, F. (2012). Rab11 Rescues Synaptic Dysfunction and Behavioural Deficits in a Drosophila Model of Huntington's Disease. Hum. Mol. Genet. 21, 2912-2922. doi:10.1093/hmg/dds117

Stepto, A., Gallo, J.-M., Shaw, C. E., and Hirth, F. (2014). Modelling C9ORF72 Hexanucleotide Repeat Expansion in Amyotrophic Lateral Sclerosis and Frontotemporal Dementia. Acta Neuropathol. 127, 377-389. doi:10.1007/ s00401-013-1235-1

Słabicki, M., Theis, M., Krastev, D. B., Samsonov, S., Mundwiller, E., Junqueira, M., et al. (2010). A Genome-Scale DNA Repair RNAi Screen Identifies SPG48 as a Novel Gene Associated with Hereditary Spastic Paraplegia. Plos Biol. 8, e1000408. doi:10.1371/journal.pbio.1000408

Taylor, J. P., Brown, R. H., and Cleveland, D. W. (2016). Decoding ALS: from Genes to Mechanism. Nature 539, 197-206. doi:10.1038/nature20413

Trojsi, F., D'Alvano, G., Bonavita, S., and Tedeschi, G. (2020). Genetics and Sex in the Pathogenesis of Amyotrophic Lateral Sclerosis (ALS): Is There a Link? Int. J. Mol. Sci. 21 (10), 3647. doi:10.3390/ijms21103647

Valdmanis, P. N., Meijer, I. A., Reynolds, A., Lei, A., MacLeod, P., Schlesinger, D., et al. (2007). Mutations in the KIAA0196 Gene at the SPG8 Locus Cause Hereditary Spastic Paraplegia. Am. J. Hum. Genet. 80, 152-161. doi:10.1086/ 510782

Waite, A. J., Bäumer, D., East, S., Neal, J., Morris, H. R., Ansorge, O., et al. (2014). Reduced C9orf72 Protein Levels in Frontal Cortex of Amyotrophic Lateral Sclerosis and Frontotemporal Degeneration Brain with the C9ORF72 Hexanucleotide Repeat Expansion. Neurobiol. Aging 35, e5. doi:10.1016/ j.neurobiolaging.2014.01.016

Wojnacki, J., and Galli, T. (2016). Membrane Traffic during Axon Development. Devel Neurobio. 76, 1185-1200. doi:10.1002/dneu.22390

Yang, Y., Hentati, A., Deng, H.-X., Dabbagh, O., Sasaki, T., Hirano, M., et al. (2001). The Gene Encoding Alsin, a Protein with Three Guanine-Nucleotide Exchange Factor Domains, Is Mutated in a Form of Recessive Amyotrophic Lateral Sclerosis. Nat. Genet. 29, 160-165. doi:10.1038/ng1001-160

Zárate, S., Stevnsner, T., and Gredilla, R. (2017). Role of Estrogen and Other Sex Hormones in Brain Aging. Neuroprotection and DNA Repair. Front. Aging Neurosci. 9, 430. doi:10.3389/fnagi.2017.00430

Zhang, J., Su, G., Wu, Q., Liu, J., Tian, Y., Liu, X., et al. (2020). Rab11-mediated Recycling Endosome Role in Nervous System Development and Neurodegenerative Diseases. Int. J. Neurosci., 1-7. doi:10.1080/ 00207454.2020.1761354

Zivony-Elboum, Y., Westbroek, W., Kfir, N., Savitzki, D., Shoval, Y., Bloom, A., et al. (2012). A Founder Mutation in Vps37A Causes Autosomal Recessive Complex Hereditary Spastic Paraparesis. J. Med. Genet. 49, 462-472. doi:10.1136/jmedgenet-2012-100742

Conflict of Interest: The authors declare that the research was conducted in the absence of any commercial or financial relationships that could be construed as a potential conflict of interest.

Publisher's Note: All claims expressed in this article are solely those of the authors and do not necessarily represent those of their affiliated organizations, or those of the publisher, the editors and the reviewers. Any product that may be evaluated in this article, or claim that may be made by its manufacturer, is not guaranteed or endorsed by the publisher.

Copyright (C) 2021 Wilkinson, Starke and Barbee. This is an open-access article distributed under the terms of the Creative Commons Attribution License (CC BY). The use, distribution or reproduction in other forums is permitted, provided the original author(s) and the copyright owner(s) are credited and that the original publication in this journal is cited, in accordance with accepted academic practice. No use, distribution or reproduction is permitted which does not comply with these terms. 